This article was retracted on January 29, 2018 (J. Am. Chem. Soc. 2018, DOI: 10.1021/jacs.7b08106).

\title{
Mutational Analysis of 48G7 Reveals that Somatic Hypermutation Affects Both Antibody Stability and Binding Affinity
}

\author{
Sophie B. Sun,† Shiladitya Sen, $\$$ Nam-Jung Kim, † Thomas J. Magliery, ${ }^{*}, \$$ Peter G. Schultz, ${ }^{*}, \dagger$ and Feng Wang*, $\neq$ \\ †Department of Chemistry and the Skaggs Institute for Chemical Biology, The Scripps Research Institute, 10550 North Torrey Pines Road, La \\ Jolla, California 92037, United States \\ „California Institute for Biomedical Research, 11119 North Torrey Pines Road Suite 100, La Jolla, California 92037, United States
}

${ }_{\S}$ Department of Chemistry and Biochemistry, The Ohio State University, 100 West 18th Avenue, Columbus, Ohio 43210, United States

\section{Supplementary Information}

\section{Materials and methods}

\section{Cloning of 48G7 mutants}

The heavy and light chain variable regions of mature 48G7 and germline 48G7g were synthesized by GenScript USA, Inc. (Piscataway, NJ). These were amplified using oligomers purchased from Integrated DNA Technologies, Inc., (Coralville, IA) and purified with the Nucleospin ${ }^{\circledR}$ Extract II kit (Macherey-Nagel GmbH \& Co. KG; Düren, Germany) (Table S3). These variable region cassettes were then ligated into pFUSE plasmids (InvivoGen; San Diego, CA; www.invivogen.com) with the IL-2 signal sequence. All mutations were generated through site-directed mutagenesis with Pfu UltraII HotStart DNA polymerase (Agilent Technologies; Santa Clara, CA). pFUSE vectors for each heavy and light chain mutant were transformed into TOP10 E. coli cells (Life Technologies Co.; Carlsbad, CA) and grown in $200 \mathrm{~mL} 2 \mathrm{xYT}$ medium overnight at $37^{\circ} \mathrm{C}$ and $270 \mathrm{RPM}$. Plasmid was then purified using the Qiagen EndoFree Plasmid Maxi Kit (Venlo, Netherlands).

\section{Fab expression and purification}

Fab fragments were expressed using the 293 FreeStyle $^{\mathrm{TM}}$ expression system following the commercial protocol (Life Technologies). pFUSE vectors containing the 48G7 heavy and light chain Fab fragments were co-transfected into FreeStyle $^{\mathrm{TM}} 293-\mathrm{F}$ cells using 293Fectin ${ }^{\mathrm{TM}}$. 293-F cells were cultured in FreeStyle ${ }^{\mathrm{TM}} 293$ Expression Medium to a density of $10^{6}$ cells $/ \mathrm{mL} .10 \mu \mathrm{g}$ of heavy chain and $10 \mu \mathrm{g}$ of light chain pFUSE vectors were added into $1 \mathrm{~mL}$ Opti-MEM ${ }^{\otimes}$ (Life Technologies). $40 \mu \mathrm{L}$ 293Fectin was added to $1 \mathrm{~mL}$ Opti-MEM and incubated at room temperature for 5 minutes, followed by gentle mixing with the plasmid DNA diluent and incubation for an additional 30 minutes. The mixture was transferred into $20 \mathrm{~mL}$ of $293-\mathrm{F}$ cell culture and incubated at $37^{\circ} \mathrm{C}, 100 \mathrm{RPM}$, and $5 \% \mathrm{CO}_{2}$. The culture supernatant was harvested after 48 hours. Cells were resuspended in fresh media and cultured for another 48 hours for a second harvest. The supernatant was adjusted to $\mathrm{pH} 8.0$ with a solution of $2 \mathrm{M}$ Tris- $\mathrm{HCl}, \mathrm{pH} 8.0$, and then loaded onto a Ni-NTA column (Qiagen) and washed twice with 5 column volumes of wash buffer ( $50 \mathrm{mM}$ Tris- $\mathrm{HCl}, 20 \mathrm{mM}$ imidazole, 300 $\mathrm{mM} \mathrm{NaCl}, \mathrm{pH} 8.0$ ). The pure Fab fragment was eluted by 5 column volumes of elution buffer ( $50 \mathrm{mM}$ Tris- $\mathrm{HCl}, 400$ $\mathrm{mM}$ imidazole, $300 \mathrm{mM} \mathrm{NaCl}, \mathrm{pH} 8.0$ ) and then buffer-exchanged to a low salt buffer $(25 \mathrm{mM}$ Tris- $\mathrm{HCl}, 50 \mathrm{mM} \mathrm{NaCl}$, $\mathrm{pH}$ 8.0). Final yields for each mutant ranged from $10-50 \mu \mathrm{g}$ from a $20 \mathrm{~mL}$ culture.

\section{Extrinsic fluorescence assay to estimate stability (High-Throughput Thermal Scanning, HTTS)}

SYPRO $^{\text {rs }}$ Orange Protein Gel Stain (Life Technologies Co., Carlsbad, CA) was supplied in DMSO at 5000x the working concentration for PAGE staining. Spectra were obtained on a Bio-Rad CFX96 thermal cycler Real-Time Detection System. Samples with a working volume of $20 \mu \mathrm{L}$ per well were prepared by mixing $1 \mu \mathrm{L}$ of $200 \times$ SYPRO $^{\text {Tx }}$ Orange (final 
concentration $10 \mathrm{x})$ with $19 \mu \mathrm{L}$ of protein $(0.1 \mathrm{mg} / \mathrm{mL}$ or $2.1 \mu \mathrm{M})$ in low-salt buffer $(25 \mathrm{mM}$ Tris- $\mathrm{HCl}, 50 \mathrm{mM} \mathrm{NaCl}$, $\mathrm{pH}$ 8.0), loaded into Bio-Rad 96-well 0.2 thin-wall PCR plates, and sealed with optical-quality sealing tape (Bio-Rad Laboratories, Inc.; Hercules, CA). In assays testing thermal stability in the presence of hapten, JWJ1 was added at a final concentration of $1 \mathrm{mM}$, which should saturate the binding sites for all of the mutants since their measured $K_{\mathrm{D}}$ values are well below millimolar range. Thermal denaturations (ramp rate of $1{ }^{\circ} \mathrm{C} / \mathrm{min}$ at $0.2^{\circ} \mathrm{C}$ intervals with an equilibration of 5 seconds at each temperature prior to measurement) were acquired by measuring fluorescence intensities using the FRET channel with excitation from $450-490 \mathrm{~nm}$ and detection from $560-580 \mathrm{~nm}$ (also referred to as differential scanning fluorimetry, DSF). All data were exported and plotted in Microsoft Excel 2010.

\section{Binding affinity measurements}

$K_{\mathrm{D}}$ values were measured with bio-layer interferometry technology on the Octet RED96 system (ForteBio, Inc.; Menlo Park, CA). Biotinylated JWJ1 hapten was dissolved at a concentration of $10 \mu \mathrm{g} / \mathrm{mL}$ in $1 \mathrm{x}$ kinetics buffer (1xPBS $\mathrm{pH} 7.4$, 0.01\% BSA, $0.002 \%$ Tween 20) for immobilization on Dip and Read ${ }^{\mathrm{TM}}$ Streptavidin biosensors (ForteBio). All binding data were collected at $30{ }^{\circ} \mathrm{C}$. Each $48 \mathrm{G} 7$ mutant Fab was first analyzed at $100 \mathrm{nM}$ in a single-pass analysis. Then, 4-5 concentrations of each mutant were used in triplicate, with the highest concentration adjusted to the measured $K_{\mathrm{D}}$ in the single-pass analysis and then serially diluted down 2-fold for the lower concentrations. Baseline, wash, and dissociation steps were performed in $1 \mathrm{x}$ kinetics buffer alone. The $K_{\mathrm{D}}$ values reported in this study were calculated from the ratio of $\mathrm{k}_{\text {off }}$ to $\mathrm{k}_{\mathrm{on}}$.

\section{Differential scanning calorimetry}

Differential scanning calorimetry (DSC) measurements were performed using a VP-Capillary DSC system (MicroCal, Inc.; Piscataway, NJ). The antibody concentrations were adjusted to $0.3 \mathrm{mg} / \mathrm{mL}$ prior to the measurement. The corresponding buffer $(25 \mathrm{mM}$ Tris- $\mathrm{HCl}, 50 \mathrm{mM} \mathrm{NaCl}, \mathrm{pH} 8.0)$ was used as a reference. The samples were heated from $14^{\circ} \mathrm{C}$ to $95^{\circ} \mathrm{C}$ at a rate of $1{ }^{\circ} \mathrm{C} / \mathrm{min}$ after initial 8 minutes of equilibration at $14^{\circ} \mathrm{C}$. A filtering period of 10 seconds was used and data were analyzed using Origin 7.0 software (OriginLab Corporation; Northampton, MA). Thermograms were corrected by subtraction of buffer-only scans and then normalized to the molar concentration of the protein. The final excess heat-capacity thermogram was obtained by interpolating a cubic baseline in the transition region. The midpoint of a thermal transition temperature $\left(T_{\mathrm{m}}\right)$ was obtained by analyzing the data using Origin 7.0 software provided with the instrument and using a two state non- $\Delta C_{\mathrm{p}}$ fit.

\section{Conjugation of JWJ1 hapten to biotinylated linker}

To a solution of JWJ1 phosphonate $\mathbf{1}^{1}(17 \mathrm{mg}, 0.057 \mathrm{mmol})$ in DMF $(1 \mathrm{~mL})$ was added diisopropylethylamine $(13 \mu \mathrm{L}$, $0.074 \mathrm{mmol})$ and HATU $(26 \mathrm{mg}, 0.068 \mathrm{mmol})$ at $0{ }^{\circ} \mathrm{C}$. The reaction mixture was stirred for 20 min, followed by addition of commercially available EZ-Link Amine-PEG - -Biotin (Thermo Fisher Scientific, Inc.; Rockford, IL) at $0{ }^{\circ} \mathrm{C}$. The reaction mixture was stirred for an additional 2 hours and then quenched with saturated aqueous $\mathrm{NH}_{4} \mathrm{Cl}$ and concentrated in vacuo. The residue was purified by HPLC on an Agilent Prep C-18 reverse phase column, $10 \mathrm{~mm}$ X $250 \mathrm{~mm}\left(\mathrm{CH}_{3} \mathrm{CN}\right.$ with $0.035 \%$ TFA: $\mathrm{H}_{2} \mathrm{O}$ with $0.05 \% \mathrm{TFA}=98: 2$ to $2: 98$, Running time: $30 \mathrm{~min}, 30 \mathrm{~mL} / \mathrm{min}, 254 \mathrm{~nm}$, retention time $25.7 \mathrm{~min}$ ). $\mathrm{CH}_{3} \mathrm{CN}$, TFA and $\mathrm{H}_{2} \mathrm{O}$ were removed in vacuo to give compound 3 . ( $12 \mathrm{mg}, 0.018 \mathrm{mmol}, 32 \%$ from 1). ${ }^{1} \mathrm{H}-\mathrm{NMR}\left(\mathrm{CD}_{3} \mathrm{OD}, 400 \mathrm{MHz}\right) \delta 8.28(\mathrm{~d}, 2 \mathrm{H}, J=9.1 \mathrm{~Hz}), 7.42(\mathrm{~d}, 2 \mathrm{H}, J=8.7 \mathrm{~Hz}), 4.50(\mathrm{dd}, 1 \mathrm{H}, J=7.5,4.8$ $\mathrm{Hz}), 4.31(\mathrm{dd}, 1 \mathrm{H}, J=7.8,4.5 \mathrm{~Hz}), 3.61(\mathrm{~s}, 4 \mathrm{H}), 3.54(\mathrm{~m}, 4 \mathrm{H}), 3.36(\mathrm{~m}, 4 \mathrm{H}), 3.21(\mathrm{~m}, 1 \mathrm{H}), 2.93(\mathrm{ddd}, 1 \mathrm{H}, J=12.8,5.0$, $2.2 \mathrm{~Hz}), 2.71(\mathrm{~d}, 1 \mathrm{H}, \mathrm{J}=12.7 \mathrm{~Hz}), 2.30-2.17(\mathrm{~m}, 4 \mathrm{H}), 2.07-1.90(\mathrm{~m}, 2 \mathrm{H}), 1.75-1.42(\mathrm{~m}, 10 \mathrm{H})$.

LR-MS (FAB) $m / z\left(\mathrm{M}+\mathrm{H}^{+}\right) 660$.

HR-MS (ESI-TOF) calculated for $\left(\mathrm{M}+\mathrm{H}^{+}\right)$660.2463; found 660.2465 
<smiles>[CH]1C2CC1C2</smiles> 


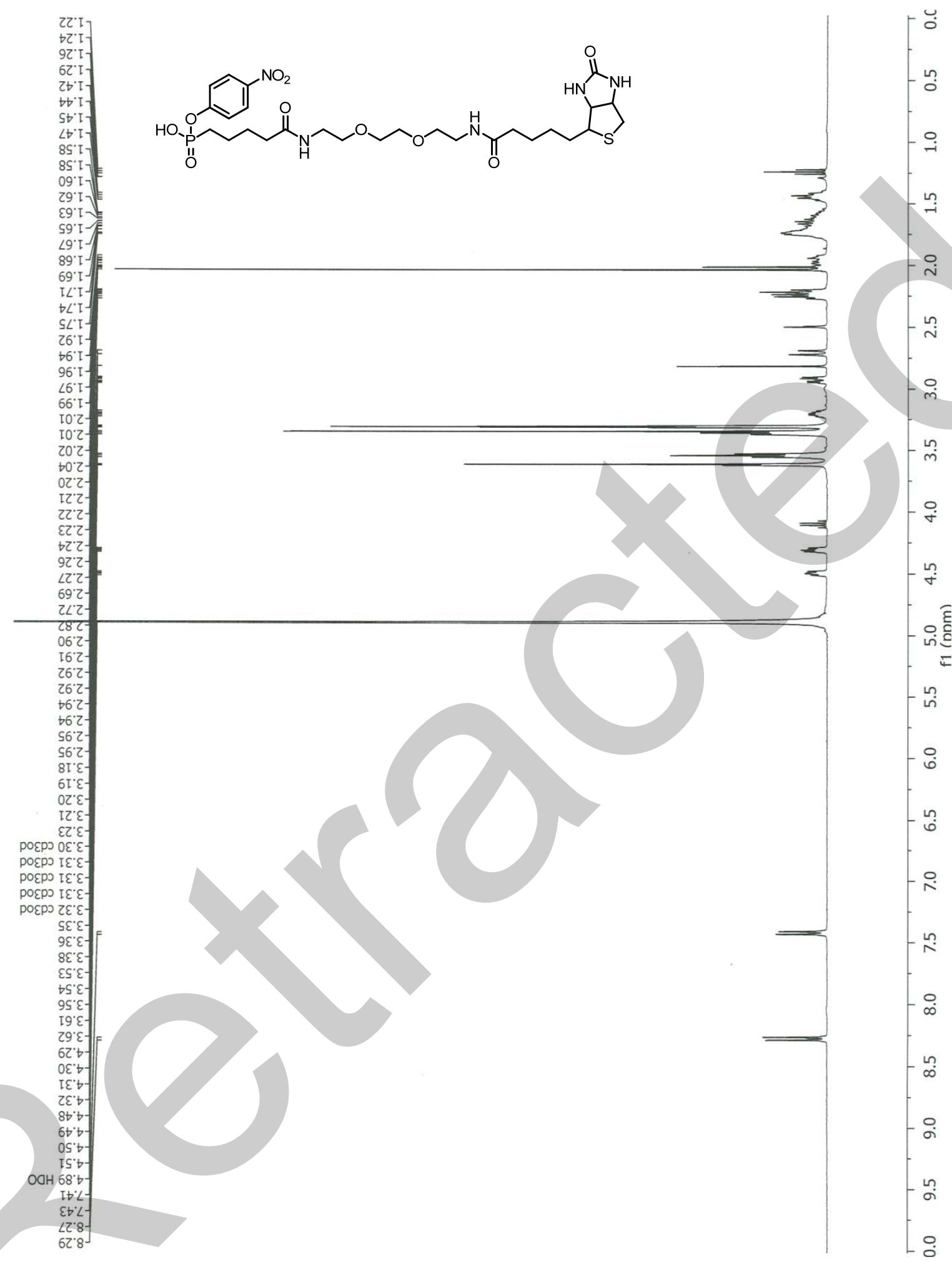

Figure S1. ${ }^{1} \mathrm{H}-\mathrm{NMR}$ (Methanol- $d_{4}, 400 \mathrm{MHz}$ ) of JWJ1-biotin conjugation

4 


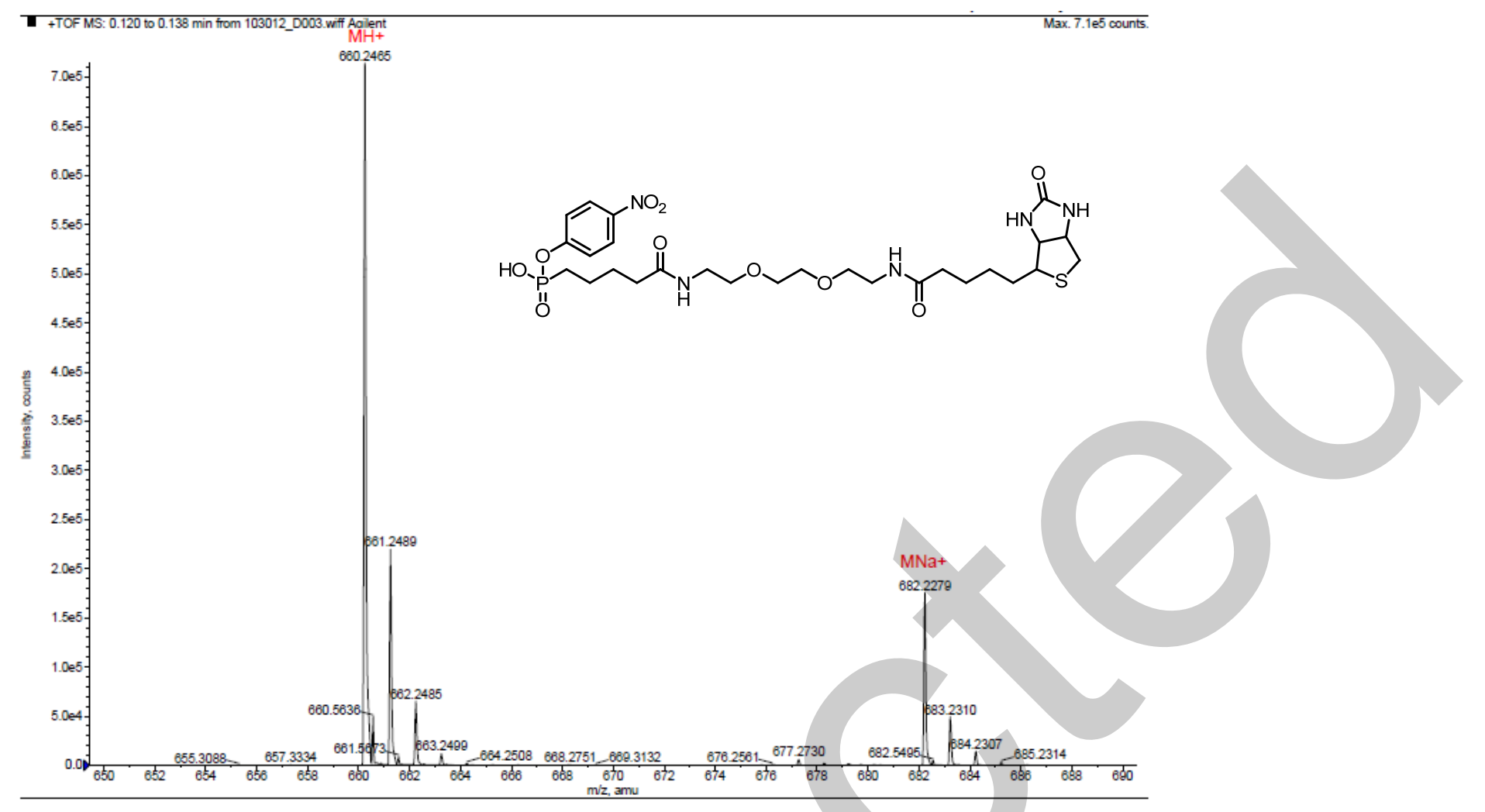

Figure S2. ESI-TOF MS of JWJ1-biotin conjugate

(A) Heavy chain

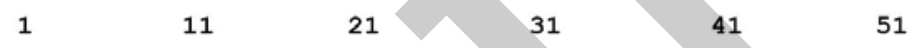

48G7g EVQLQQSGAELVKPGASVKLSCTAS GFNIKDTYMH WVKQRPEQGLEWIG RIDPANGNTKY

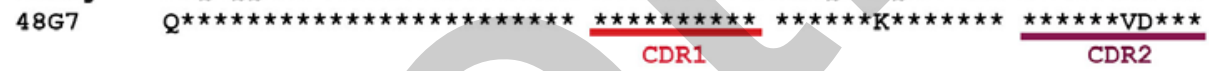

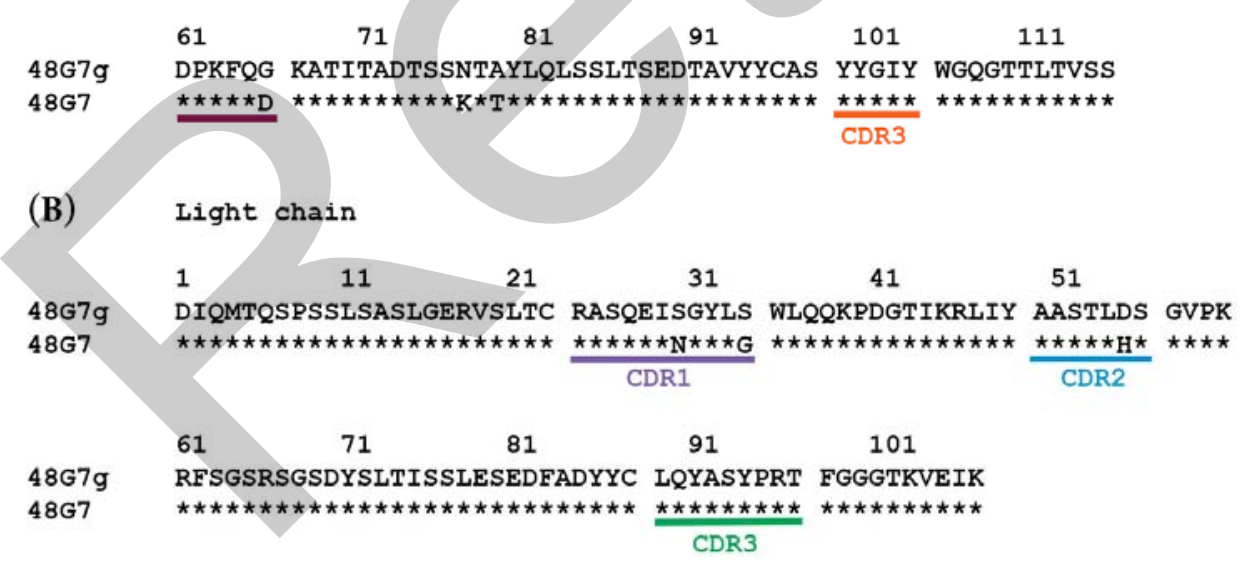

Figure S3. Sequence alignment of germline 48G7g with mature $48 \mathrm{G} 7$ (A) heavy chain and (B) light chain variable regions. Complementarity determining regions (CDRs) are underlined in color. 

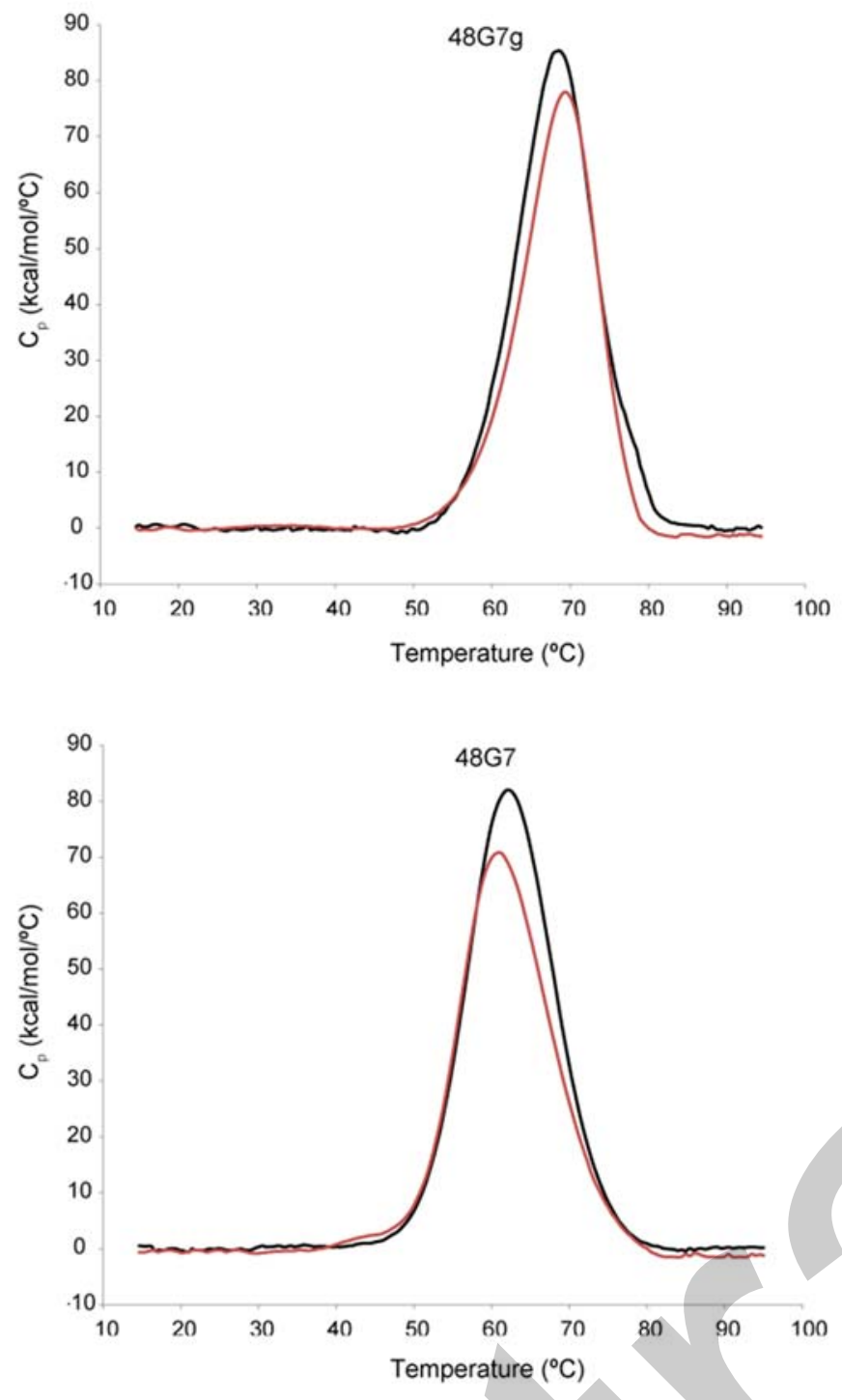

\begin{tabular}{|c|c|c|c|c|}
\hline & DSF $T_{\mathrm{m}}$ & Std error & DSC $T_{\mathrm{m}}$ & Std error \\
\hline 48G7g & 70.5 & 0.1 & 69.4 & 0.4 \\
\hline $48 \mathrm{G} 7$ & 63.5 & 0.1 & 62.2 & 0.6 \\
\hline 48G7b & 52 & 0.2 & 51.3 & 0.4 \\
\hline
\end{tabular}

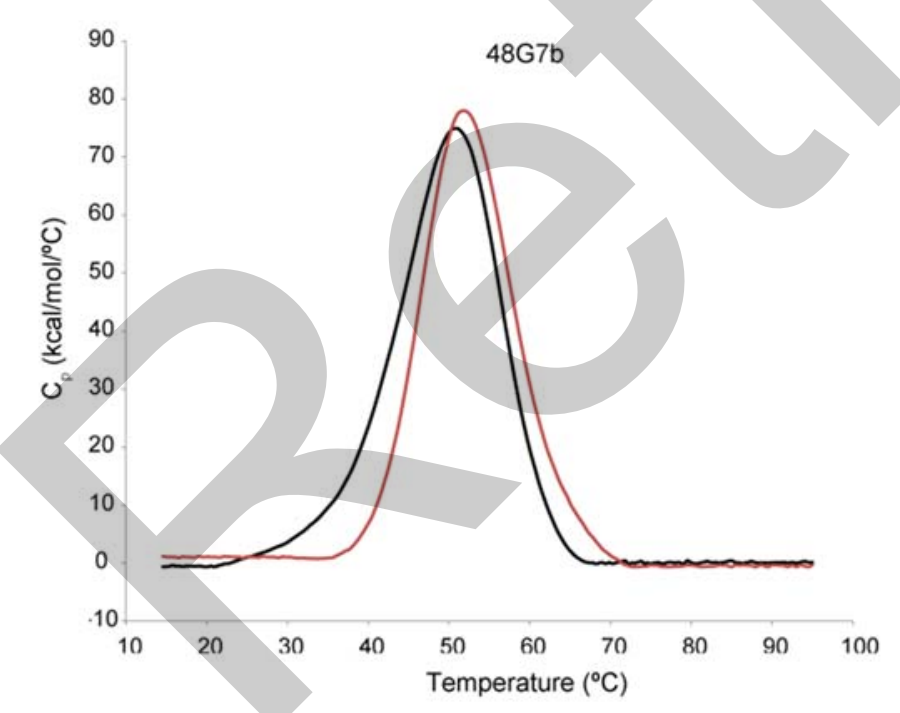

Figure S4. Differential scanning calorimetry (DSC) curves and comparison against differential scanning fluorimetry (DSF) values for 48G7g, 48G7, and 48G7b. 

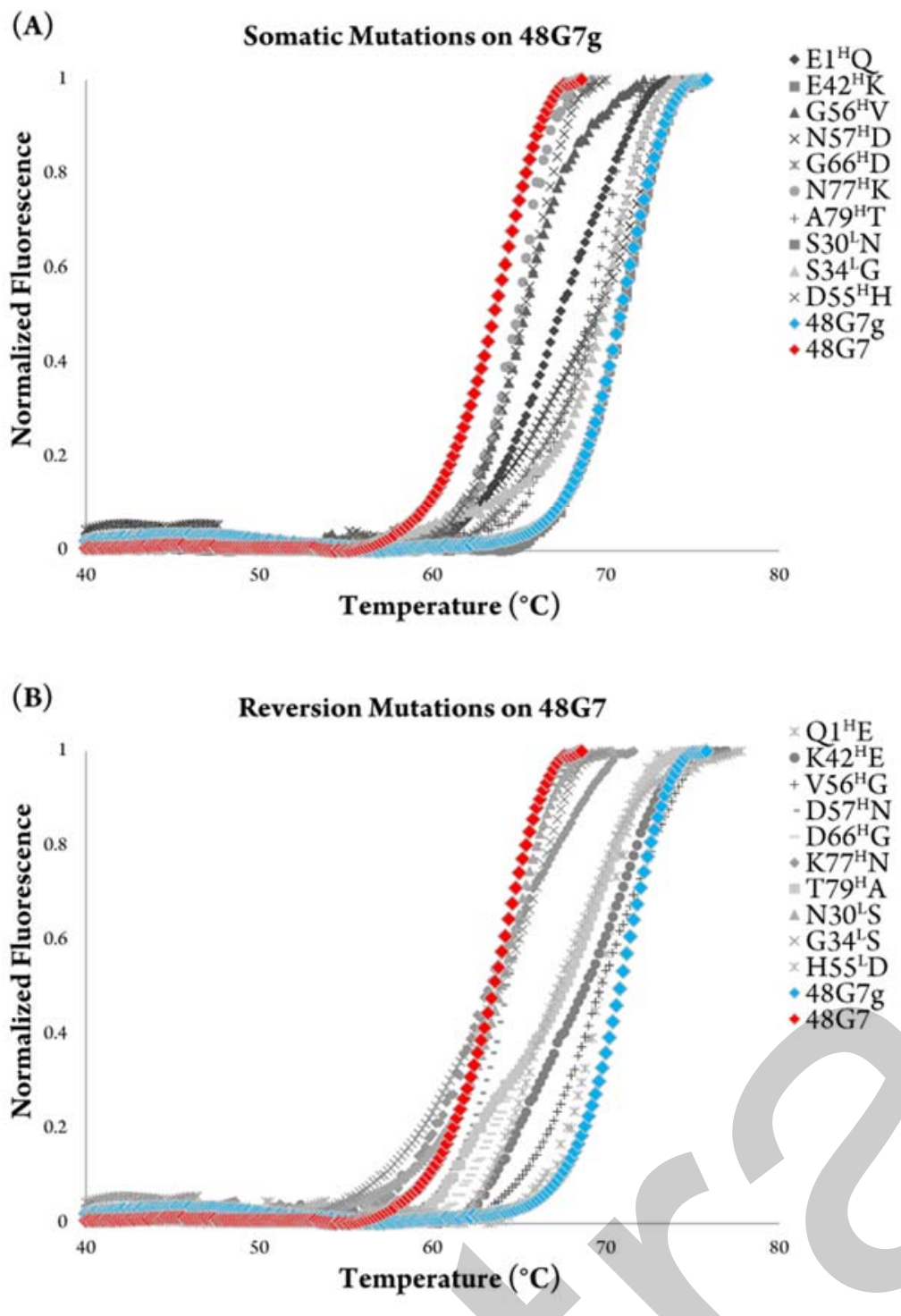

$\mathrm{Q} 1^{\mathrm{H}} \mathrm{E}$ - $\mathrm{K} 42^{\mathrm{H}} \mathrm{E}$

- D $57^{\mathrm{H}} \mathrm{N}$

D $66^{\mathrm{H}} \mathrm{G}$

- $\mathrm{K} 77^{\mathrm{H}} \mathrm{N}$

- T79 ${ }^{\mathrm{H}} \mathrm{A}$

${ }_{\triangle} \mathrm{N} 30^{\mathrm{L}} \mathrm{S}$

G34 ${ }^{\mathrm{L}} \mathrm{S}$

$\times 55^{2} \mathrm{D}$

-48G7g

+48G7

(C) Combined Mutations on 48G7b

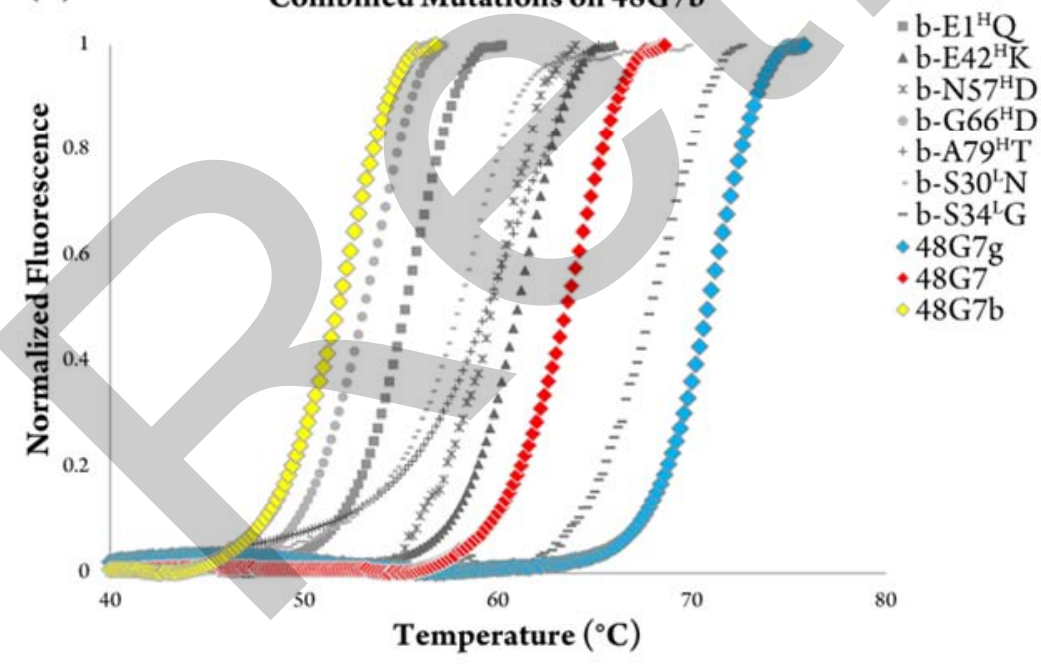

Figure S5. Sample thermal denaturation curves for mutants of 48G7. A lowercase ' $b$ ' indicates that mutations that follow have been added to the binding mutant $48 \mathrm{G} 7 \mathrm{~b}$. 


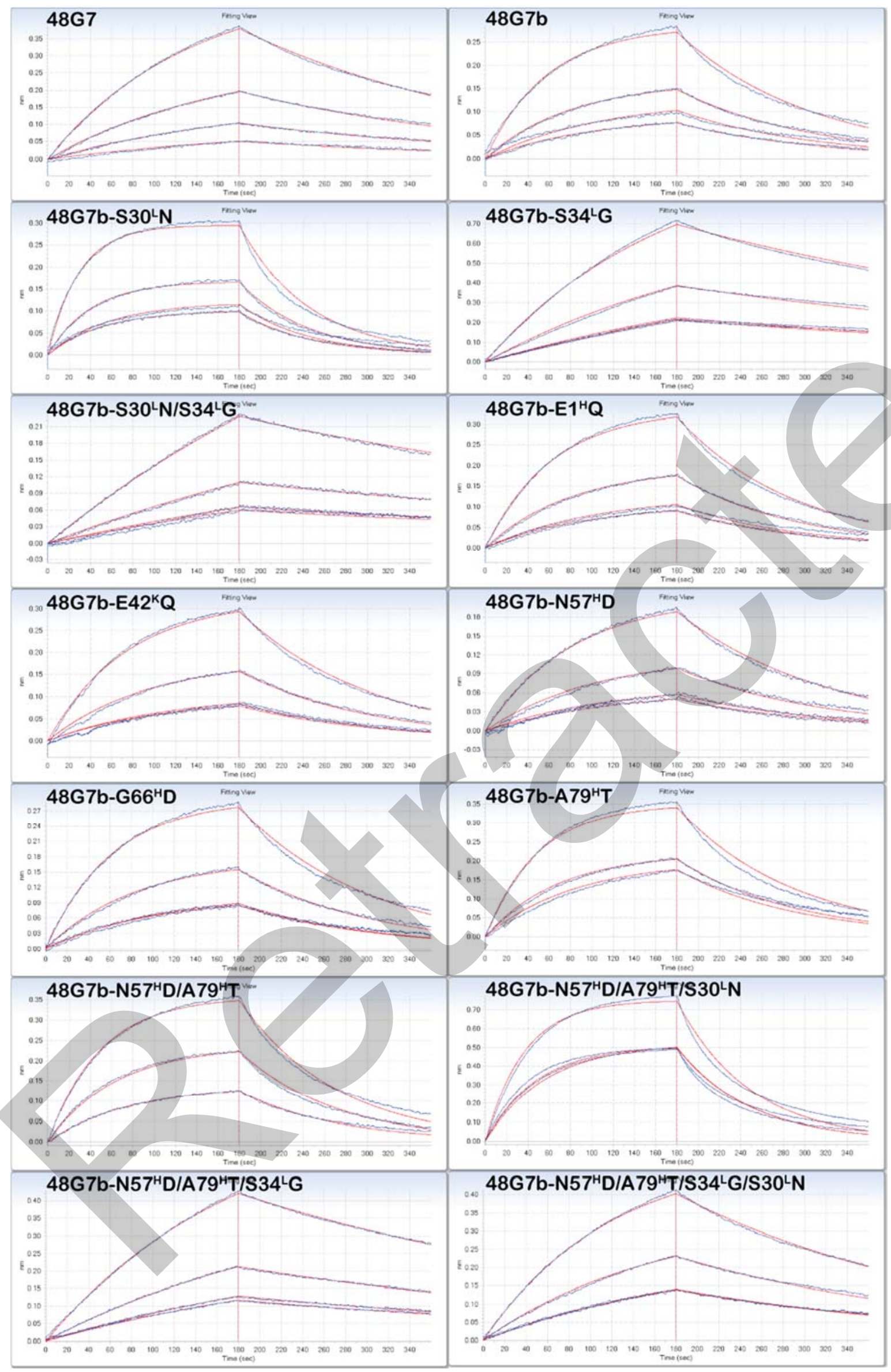

Figure S6. Sample binding curves used to determine $K_{\mathrm{D}}$ values 
$\underline{\operatorname{Tm}\left({ }^{\circ} \mathrm{C}\right)}$

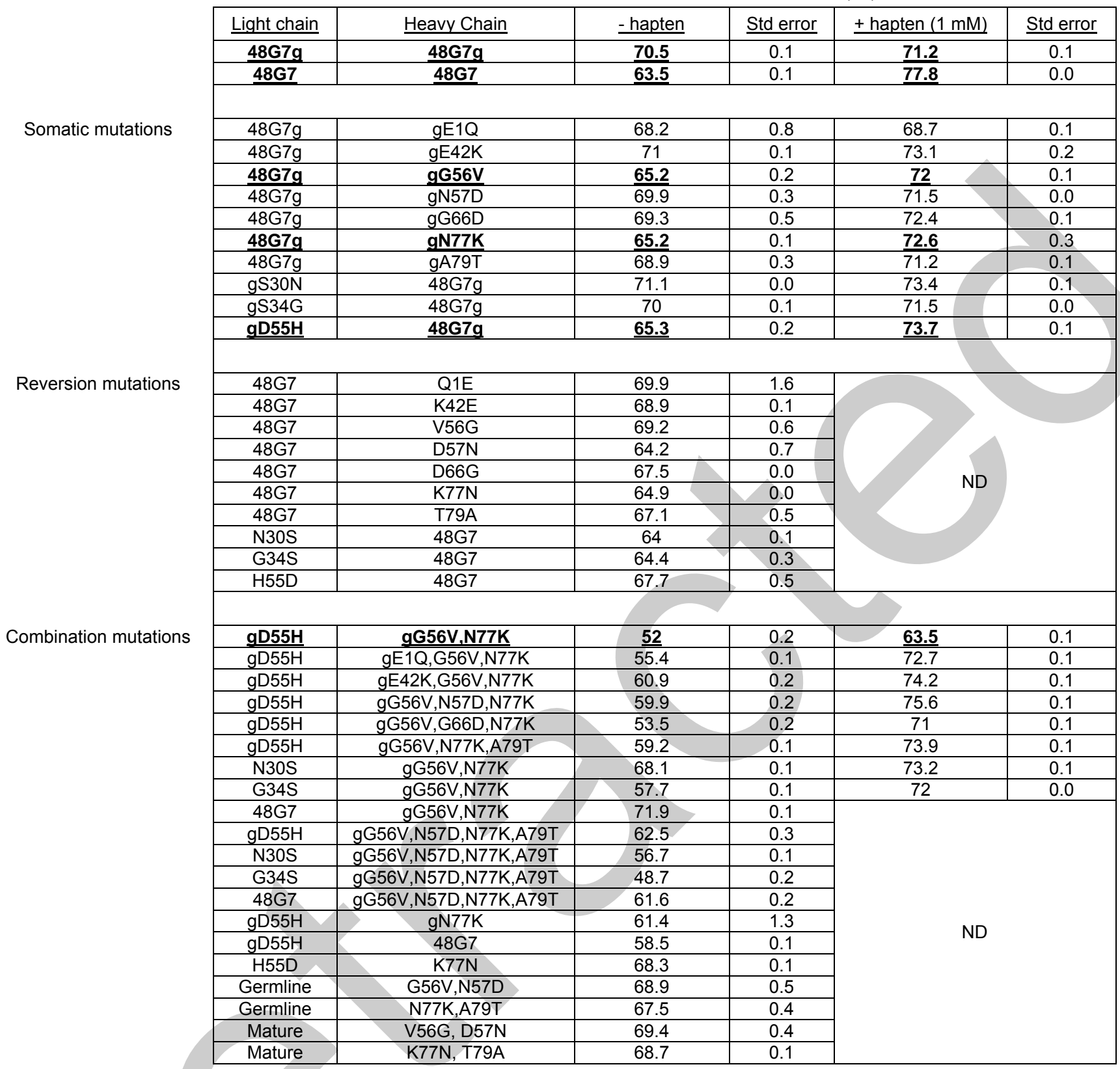

Table S1. Melting temperatures for mutants obtained through DSF. Notable reference points are highlighted in bold. A lowercase ' $\mathrm{g}$ ' indicates that the mutations that follow are somatic mutations added to 48G7g. "ND" - not determined 


\begin{tabular}{|c|c|c|c|c|}
\hline \multicolumn{4}{|c|}{ Primers to clone $48 \mathrm{G} 7$ into pFUSE vectors for Fab expression } & \multirow[t]{2}{*}{ Notes } \\
\hline $48 \mathrm{G} 7$ & Heavy chain & Forward & acttgtcacgaattcgcaggtgcagctgcagcAGTCTGGGGCAGAGCTTGTG & \\
\hline & & Reverse & gtctggccagctagcacttattagtgatgatgatggtgatggctgccgtcACAAGATTTGGGCTCAACTTTCTTGTCC & Adds His6 tag \\
\hline & Light chain & Forward & acttgtcacgaattcgGATATCCAGATGACCCAGTCTCCATCC & Germline generated from mature \\
\hline & & Reverse & gtctggccagctagcacttattaacactctcccctgttgaagctctttgtgacgggCGAGCTCAGGCCCTGATGG & \\
\hline 48G7g & Heavy chain & Forward & acttgtcacgaattcgGAGGTGCAGCTGCAGCAG & Reverse primer same as for mature \\
\hline \multicolumn{5}{|c|}{ Primers for site-directed mutagenesis to generate $48 \mathrm{G} 7$ mutants } \\
\hline \multirow[t]{18}{*}{ 48G7g } & $\mathrm{E} 1^{\mathrm{H}} \mathrm{Q}$ & Forward & CACTAAGTCTTGCACTTGTCACGAATTCGCAGGTGCAG & \\
\hline & & Reverse & GACTGCTGCAGCTGCACCTGCGAATTCG & \\
\hline & $\mathrm{E} 42^{\mathrm{H}} \mathrm{K}$ & Forward & CTGGGTGAAGCAGAGGCCTAAACAGGGC & \\
\hline & & Reverse & CAATCCACTCCAGGCCCTGTTTAGGCCTCT & 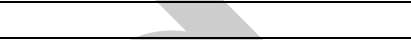 \\
\hline & $\mathrm{G}^{2} 6^{\mathrm{H}} \mathrm{V}$ & Forward & GGATTGGAAGGATTGATCCTGCGAATGTTAATACTA & \\
\hline & & Reverse & CTTGCCCTGGAACTTCGGGTCATATTTAGTATTAACATTCGC & \\
\hline & $\mathrm{N} 57^{\mathrm{H}} \mathrm{D}$ & Forward & GATTGGAAGGATTGATCCTGCGAATGGTGATACTAAA & \\
\hline & & Reverse & CCTGGAACTTCGGGTCATATTTAGTATCACCATTC & \\
\hline & G66 ${ }^{\mathrm{H} D}$ & Forward & ATACTAAATATGACCCGAAGTTCCAGGACAAGGCCA & 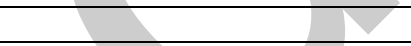 \\
\hline & & Reverse & GAGGATGTATCTGCTGTTATAGTGGCCTTGTCCTGGAAC & \\
\hline & $\mathrm{N} 77^{\mathrm{H}} \mathrm{K}$ & Forward & GCCACTATAACAGCAGATACATCCTCCAAGACAGCCT & \\
\hline & & Reverse & CTGAGCTGCAGGTAGGCTGTCTTGGAGG & - \\
\hline & $\mathrm{A} 79^{\mathrm{H}} \mathrm{T}$ & Forward & CCACTATAACAGCAGATACATCCTCCAACACAACCTACCT & \\
\hline & & Reverse & GCTGCTGAGCTGCAGGTAGGTTGTGTTG & 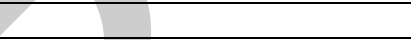 \\
\hline & $\mathrm{G} 56^{\mathrm{H}} \mathrm{V}, \mathrm{N} 57^{\mathrm{H}} \mathrm{D}$ & Forward & GGATTGGAAGGATTGATCCTGCGAATGTTGATACTAAA & \\
\hline & & Reverse & CCTGGAACTTCGGGTCATATTTAGTATCAACATTCGCA & \\
\hline & $\mathrm{N} 77^{\mathrm{H}} \mathrm{K}, \mathrm{A} 79^{\mathrm{H}} \mathrm{T}$ & Forward & GCCACTATAACAGCAGATACATCCTCCAAGACAACCTACCTG & 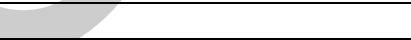 \\
\hline & & Reverse & GCTGCTGAGCTGCAGGTAGGTTGTCTTGGAGGA & \\
\hline \multirow[t]{24}{*}{$48 \mathrm{G} 7$} & $\mathrm{Q} 1^{\mathrm{H}} \mathrm{E}$ & Forward & CACTAAGTCTTGCACTTGTCACGAATTCGGAGGTGCAG & \\
\hline & & Reverse & GACTGCTGCAGCTGCACCTCCGAATTCG & \\
\hline & $\mathrm{K} 42^{\mathrm{H}} \mathrm{E}$ & Forward & CTGGGTGAAGCAGAGGCCTGAACAGGGC & \\
\hline & & Reverse & CAATCCACTCCAGGCCCTGTTCAGGCCTCT & \\
\hline & $\mathrm{V} 56^{\mathrm{H} G}$ & Forward & GGATTGGAAGGATTGATCCTGCGAATGGTGATACTA & \\
\hline & & Reverse & CTTGTCCTGGAACTTCGGGTCATATTTAGTATCACCATTCGCA & \\
\hline & $\mathrm{D} 57^{\mathrm{H}} \mathrm{N}$ & Forward & GATTGGAAGGATTGATCCTGCGAATGTTAATACTAAA & \\
\hline & & Reverse & CCTTGTCCTGGAACTTCGGGTCATATTTAGTATTAACATTCG & \\
\hline & $\mathrm{D} 66^{\mathrm{H} G}$ & Forward & ATACTAAATATGACCCGAAGTTCCAGGGCAAGGCCA & \\
\hline & & Reverse & GAGGATGTATCTGCTGTTATAGTGGCCTTGCCCTGGAAC & \\
\hline & $\mathrm{K} 77^{\mathrm{H}} \mathrm{N}$ & Forward & GCCACTATAACAGCAGATACATCCTCCAACACAACCTA & \\
\hline & & Reverse & GCTGAGCTGCAGGTAGGTTGTGTTGGAGGA & \\
\hline & $\mathrm{T}^{2} 9^{\mathrm{H}} \mathrm{A}$ & Forward & CACTATAACAGCAGATACATCCTCCAAGACAGCCTACCTG & \\
\hline & & Reverse & GCTGCTGAGCTGCAGGTAGGCTGTCTTGG & \\
\hline & $\mathrm{V} 56^{\mathrm{H}} \mathrm{G}, \mathrm{D} 57^{\mathrm{H}} \mathrm{N}$ & Forward & GGATTGGAAGGATTGATCCTGCGAATGGTAATACTAAA & \\
\hline & & Reverse & CCTTGTCCTGGAACTTCGGGTCATATTTAGTATTACCATTCGCA & \\
\hline & $\mathrm{K} 77^{\mathrm{H}} \mathrm{N}, \mathrm{T} 79^{\mathrm{H}} \mathrm{A}$ & Forward & GCCACTATAACAGCAGATACATCCTCCAACACAGCCTACCTG & \\
\hline & & Reverse & GCTGCTGAGCTGCAGGTAGGCTGTGTTGGAGGA & \\
\hline & $\mathrm{N} 30^{\mathrm{L} S}$ & Forward & CACTTGTCGGGCAAGTCAGGAAATTAGTGGTTACTTAGGC & \\
\hline & & Reverse & CTGCTGAAGCCAGCCTAAGTAACCACTAATTTCCTGACT & \\
\hline & $\mathrm{H} 55^{\mathrm{L}} \mathrm{D}$ & Forward & CCTGATCTACGCCGCATCCACTTTAGATTCTGG & \\
\hline & & Reverse & CCACTGAACCTTTTTGGGACACCAGAATCTAAAGTG & \\
\hline & G34'S & Forward & CGGGCAAGTCAGGAAATTAATGGTTACTTAAGCTGGCT & \\
\hline & 2 & Reverse & CATCTGGTTTCTGCTGAAGCCAGCTTAAGTAAC & \\
\hline
\end{tabular}

Table S3. Primers used to clone 48G7 into pFUSE vectors and to perform site-directed mutagenesis to produce $48 \mathrm{G} 7$ mutants 


\begin{tabular}{|c|c|c|c|}
\hline Light Chain & Heavy Chain & Expected Mass & ESI Result \\
\hline Germline & Germline & 47557 & 47558 \\
\hline Germline & E1Q & 47540 & 47543 \\
\hline Germline & $\mathrm{E} 42 \mathrm{~K}$ & 47556 & 47560 \\
\hline Germline & G56V & 47599 & 47604 \\
\hline Germline & N57D & 47558 & 47563 \\
\hline Germline & G66D & 47615 & 47620 \\
\hline Germline & N77K & 47571 & 47576 \\
\hline Germline & A79T & 47587 & 49900* \\
\hline Germline & G56V,N57D & 47600 & 47606 \\
\hline Germline & N77K,A79T & 47601 & 47606 \\
\hline Mature & Mature & 47703 & 47703 \\
\hline S30N & Germline & 47584 & 47588 \\
\hline S34G & Germline & 47527 & 47532 \\
\hline D55H & Germline & 47579 & 47584 \\
\hline N30S & Mature & 47676 & 47681 \\
\hline G34S & Mature & 47733 & 47737 \\
\hline H55D & Mature & 47681 & 47685 \\
\hline Mature & Q1E & 47720 & 47723 \\
\hline Mature & $\mathrm{K} 42 \mathrm{E}$ & 47704 & 47706 \\
\hline Mature & V56G & 47661 & 47663 \\
\hline Mature & D57N & 47702 & 47704 \\
\hline Mature & D66G & 47645 & 47646 \\
\hline Mature & K77N & 47689 & 49943 \\
\hline Mature & T79A & 47673 & 47675 \\
\hline Mature & V56G, D57N & 47660 & 47662 \\
\hline Mature & K77N, T79A & 47659 & 47663 \\
\hline N30S & gG56V,N77K & 47605 & 47605 \\
\hline G34S & gG56V,N77K & 47662 & 47662 \\
\hline Mature & gG56V,N77K & 47632 & 47632 \\
\hline gD55H & gG56V,N77K & 47635 & 47636 \\
\hline gD55H & gE1Q,G56V,N77K & 47618 & 47619 \\
\hline gD55H & gE42K,G56V,N77K & 47634 & 47636 \\
\hline gD55H & gG56V,N57D,N77K & 47636 & 47638 \\
\hline gD55H & gG56V,G66D,N77K & 47693 & 47695 \\
\hline gD55H & gG56V,N77K,A79T & 47665 & 47667 \\
\hline gD55H & gG56V,N57D,N77K,A79T & 47666 & 47668 \\
\hline N30S & gG56V,N57D,N77K,A79T & 47636 & 47637 \\
\hline G34S & gG56V,N57D,N77K,A79T & 47693 & 47694 \\
\hline Mature & gG56V,N57D,N77K,A79T & 47663 & 47664 \\
\hline gD55H & gN77K & 47593 & 47598 \\
\hline gD55H & Mature & 47706 & 47706 \\
\hline H55D & K77N & 47667 & $49913^{*}$ \\
\hline
\end{tabular}

Table S4. ESI-TOF MS data. The N-terminal glutamines cyclize, reducing the mass by $16 \mathrm{Da} .{ }^{*}$ Again, the combination of both N77 and T79 introduces a glycosylation site, which results in a higher mass (NetNGlyc 1.0 Server, http://www.cb.dtu.dk/services/NetNGlyc). 